\title{
CONVEX FUNCTIONS ON THE HEISENBERG GROUP
}

\author{
GUOZHEN LU, JUAN J. MANFREDI, AND BIANCA STROFFOLINI
}

\begin{abstract}
Convex functions in Euclidean space can be characterized as universal viscosity subsolutions of all homogeneous fully nonlinear second order elliptic partial differential equations. This is the starting point we have chosen for a theory of convex functions on the Heisenberg group.
\end{abstract}

\section{INTRODUCTION}

In the past decade research in fully nonlinear equations in Euclidean spaces has made considerable progress. We refer to two monographs in this direction by Caffarelli-Cabré [CC1] and Gutierrez [G]. An important example is the so-called Monge-Ampère equation

$$
\operatorname{det}\left(D^{2} u\right)=f .
$$

In considering solutions to the above equation convex functions play a crucial role. The notion of generalized weak solution, introduced by A.D. Aleksandrov, relies on the properties of convex functions.

Motivated by the role that convex functions play in the theory of fully nonlinear equations, we formulate several notions of convexity in the Heisenberg group toward the aim of developing an intrinsic theory of subelliptic fully nonlinear equations, see $[\mathrm{M}]$. We will discuss the notion of group convexity, horizontal convexity and viscosity convexity. The advantage of this last definition is that it can be considered even in the general case of Hörmander vector fields where no group operation exists. Our proofs are based on the viscosity theory for subelliptic equations and the geometric role played by the Carnot-Carathéodory metric. This metric is used to build "subelliptic cones."

The uniqueness theorem for viscosity solutions of second order subelliptic equations, including the subelliptic $\infty$-Laplacian was recently proved by Bieske ([B], [M]) in the case of the Heisenberg group. This result provides evidence for a fully nonlinear subelliptic theory.

Here is the plan of the paper. In section $\S 2$ we review the Euclidean case. In section $\S 3$ we study convex functions defined by requiring that their symmetrized horizontal second derivatives are non-negative in the viscosity sense. We call these functions v-convex. The main result of this section is to establish their local Lipschitz continuity. In section $\S 4$ we will present the notion of horizontal convexity, which we believe has many interesting properties. This notion is quite natural

1991 Mathematics Subject Classification. Primary: 49L25, 35J70; Secondary: 35J67, 22E30.

Key words and phrases. horizontal convexity, viscosity convexity, Carnot groups, Heisenberg group, subelliptic equations.

First author supported by US NSF grant DMS-9970352.

Second author supported by US NSF grant DMS-0100107.

Third author was supported by G.N.A.M.P.A. and by the 2002 project "Partial Differential Equations and Control Theory". 
in the stratified groups setting. Other researchers have independently studied it. Horizontal convexity is equivalent to a notion of convexity considered by Caffarelli and Cabré in [Ca]. It has also been studied in the forthcoming article [DGN]. Our methods are based on the theory of viscosity solutions of the infinite-Laplacian in the Heisenberg group and are significantly different from those in [DGN].

We will show that upper-semicontinuous horizontally convex functions are vconvex, and therefore Lipschitz continuous. At the present time we are able to show that these two notions of convexity are equivalent if the functions are assumed to be $C^{2}$ by deriving a subelliptic Taylor's formula with integral reminder. ${ }^{1}$ Another result of this section states that the symmetrized horizontal second derivatives of horizontally convex functions are signed Radon measures.

Let us briefly recall some basic facts about the Heisenberg group $\mathcal{H}$. The group operation in $\mathcal{H}=\left(\mathbb{R}^{3}, \cdot\right)$ is given by:

$$
p \cdot q=\left(x_{1}+x_{2}, y_{1}+y_{2}, z_{1}+z_{2}+\frac{1}{2}\left(x_{1} y_{2}-x_{2} y_{1}\right)\right)
$$

where $p=\left(x_{1}, y_{1}, z_{1}\right)$ and $q=\left(x_{2}, y_{2}, z_{2}\right)$. The Lie algebra $\mathfrak{h}$ is spanned by the left-invariant vector fields $X_{1}, X_{2}$ and $X_{3}$ given by

and

$$
\begin{aligned}
& X_{1}(p)=\frac{\partial}{\partial x}-\frac{y}{2} \frac{\partial}{\partial z} \\
& X_{2}(p)=\frac{\partial}{\partial y}+\frac{x}{2} \frac{\partial}{\partial z}
\end{aligned}
$$

$$
X_{3}(p)=\frac{\partial}{\partial z}=\left[X_{1}, X_{2}\right]
$$

where $p=(x, y, z) \in \mathbb{R}^{3}$. The Lie algebra $\mathfrak{h}$ admits a stratification. It decomposes as the vector space direct sum

$$
\mathfrak{h}=\mathfrak{h}_{1} \oplus \mathfrak{h}_{2},
$$

where $\mathfrak{h}_{1}$ is the subspace generated by $X_{1}$ and $X_{2}$ and $\mathfrak{h}_{2}=\left[\mathfrak{h}_{1}, \mathfrak{h}_{1}\right]$ is the onedimensional space generated by $X_{3}$. For this reason $\mathcal{H}$ is called a Carnot Group of step 2.

The group $\mathcal{H}$ has a family of dilations that are group homomorphisms, parameterized by $r>0$ and given by

$$
\delta_{r}(x, y, z)=\left(r x, r y, r^{2} z\right) .
$$

The exponential mapping takes the vector $x X_{1}+y X_{2}+z X_{3}$ in the Lie algebra $\mathfrak{h}$ to the point $(x, y, z)$ in the Lie group $\mathcal{H}$. These are called exponential coordinates of the first kind and allow us to identify vectors in $\mathfrak{h}$ with points in $\mathcal{H}$.

We denote by $\mathcal{H}_{0}$ the set of horizontal vectors of the form $h=(x, y, 0)$, which we may also think as the vectors of the form $x X_{1}+y X_{2}$. The horizontal subspace at $p \in \mathcal{H}$ is the 2-dimensional subspace that is linearly spanned by $X_{1}(p)$ and $X_{2}(p)$. With the above notations the horizontal subspace can be identified with the left translation by $p$ of $\mathcal{H}_{0}$, that is we have

$$
p \cdot \mathcal{H}_{0}=\text { Linear-span }\left\{X_{1}(p), X_{2}(p)\right\} .
$$

\footnotetext{
${ }^{1}$ In fact, with the help of Petri Juutinen we have been able to prove recently that these two notions of convexity agree for upper semi-continous functions. This will be the subject of a future publication.
} 
A horizontal curve $\gamma(t)$ is a piece-wise smooth curve whose tangent vector $\gamma^{\prime}(t)$ is in the the horizontal tangent space $(\gamma(t)) \cdot \mathcal{H}_{0}$ whenever it exists. Given two points $p$ and $q$ we consider the set of all possible horizontal curves joining these points:

$$
\Gamma(p, q)=\{\gamma \text { horizontal curve }: \gamma(0)=p, \gamma(1)=q\}
$$

This set is never empty by Chow's theorem (see for example [BR]). The CarnotCarathéodory distance is then defined as the infimum of the length of horizontal curves of the set $\Gamma$ :

$$
d_{C C}(p, q)=\inf _{\Gamma(p, q)} \int_{0}^{1}\left|\gamma^{\prime}(t)\right| d t
$$

We compute the length of a tangent vector by considering the vectors $\left\{X_{1}, X_{2}\right\}$ as an orthonormal basis of a sub-riemannian metric defined on the distribution of horizontal subspaces $p \cdot \mathcal{H}_{0}$. The Carnot-Carathéodory ball of radius $R$ centered at a point $p$ is given by

$$
B(p, R)=\left\{q \in \mathcal{H}: d_{C C}(p, q)<r\right\} .
$$

The Carnot-Carathéodory gauge is given by

$$
|p|_{C C}=d(0, p) \text {. }
$$

The Carnot-Carathéodory distance, being constructed in terms of left-invariant vector fields, is left-invariant and positively homogeneous of degree 1 on $\mathcal{H}$.

An important cone-like property of this gauge is that it solves the eikonal equation away from the vertical axis. This can be computed explicitly in the case of the Heisenberg group because explicit formulas for the Carnot-Carathéodory gauge are available, $[\mathrm{BR}]$. We take the opportunity to quote a recent result of Monti and Serra-Cassano [MSc] which is valid in general Carnot groups.

Theorem 1.1. Consider the horizontal gradient of $|p|_{C C}$ given by

$$
X\left(|p|_{C C}\right)=\left(X_{1}\left(|p|_{C C}\right), X_{2}\left(|p|_{C C}\right)\right) .
$$

Then, for a.e. $p \in \mathbb{R}^{3}$, we have

$$
\left|X\left(|p|_{C C}\right)\right|=\left(\left(X_{1}|p|_{C C}\right)^{2}+\left(X_{2}|p|_{C C}\right)^{2}\right)^{\frac{1}{2}}=1 .
$$

For a smooth function $u: \mathcal{H} \mapsto \mathbb{R}$ the horizontal gradient of $u$ at a point $p$ is the projection of the gradient of $u$ at $p$ onto the horizontal subspace $p \cdot \mathcal{H}_{0}$ and is given by

$$
D_{\mathrm{h}} u=\left(X_{1} u\right) X_{1}+\left(X_{2} u\right) X_{2} .
$$

The symmetrized horizontal second derivative matrix, denoted by $\left(D_{\mathrm{h}}^{2} u\right)^{\star}$ has entries

$$
\left(D_{\mathrm{h}}^{2} u\right)_{i j}^{\star}=\frac{1}{2}\left(X_{i} X_{j} u+X_{j} X_{i} u\right)
$$

for $i, j=1,2$. We recall that the derivative with respect to the $z$-axis plays the role of second derivative in the Taylor's expansion, see [FS].

We shall also use a smooth gauge, called the Heisenberg gauge, given by

$$
|p|_{\mathcal{H}}=\left(\left(x^{2}+y^{2}\right)^{2}+t^{2}\right)^{1 / 4} .
$$

By a simple version of the "ball-box" theorem, see for example [BR], there exists a constant $C$ such that for all $p \in \mathcal{H}$ we have

$$
\frac{1}{C}|p|_{\mathcal{H}} \leq|p|_{C C} \leq C|p|_{\mathcal{H}} .
$$


The corresponding distance

$$
d_{\mathcal{H}}(p, q)=\left|q^{-1} \cdot p\right|_{\mathcal{H}}
$$

is bi-Lipschitz equivalent to the Carnot-Carathéodory distance.

\section{Convexity in the Viscosity Sense in $\mathbb{R}^{n}$}

We reprove here some well known facts about convex functions in Euclidean space. The point being that the proofs presented here generalize, at least in principle, to the subelliptic case.

We will always assume that the upper-semicontinuous functions considered in this section are not identically $-\infty$.

Let $\Omega \subset \mathbb{R}^{n}$ be an open set. Consider a continuous function $F(x, z, p, M)$ in $\Omega \times \mathbb{R} \times \mathbb{R}^{n} \times \mathcal{S}^{n}$ that satisfies

$$
\left\{\begin{aligned}
F(x, z, p, 0) & =0 \\
F(x, z, p, M) & \leq F\left(x, z^{\prime}, p, M\right) \text { if } z \leq z^{\prime}, \text { and } \\
F(x, z, p, M) & \leq F\left(x, z, p, M^{\prime}\right) \text { if } M^{\prime} \leq M
\end{aligned}\right.
$$

The last two conditions indicate that $F$ is proper and degenerate elliptic according to the terminology of [CIL]. Here $\mathcal{S}^{n}$ denotes the class of $n \times n$ real symmetric matrices.

Un upper-semicontinuous function $u: \Omega \rightarrow \mathbb{R}$ is a viscosity subsolution of the equation

$$
F\left(x, u(x), D u(x), D^{2} u(x)=0\right.
$$

if whenever we have a $C^{2}$ function $\phi$ and a point $x_{0} \in \Omega$ such that $\phi$ touches $u$ from above at $x_{0}\left(u\left(x_{0}\right)=\phi\left(x_{0}\right)\right.$ and $u(x) \leq \phi(x)$ for $\left.x \neq x_{0}\right)$, the inequality

$$
F\left(x_{0}, \phi\left(x_{0}\right), D \phi\left(x_{0}\right), D^{2} \phi\left(x_{0}\right)\right) \leq 0
$$

holds.

This definition makes sense also for some operator valued functions $F(x, z, p, M)$. For example, we say that

$$
D^{2}(u) \geq-0
$$

in the viscosity sense, if whenever we have a $C^{2}$ function $\phi$ and a point $x_{0} \in \Omega$ such that $\phi$ touches $u$ from above at $x_{0}$, the inequality

$$
D^{2} \phi\left(x_{0}\right) \geq 0
$$

holds.

Theorem 2.1. Let $\Omega \subset \mathbb{R}^{n}$ be an open set and $u: \Omega \rightarrow \mathbb{R}$ be an upper-semicontinuous function. Then, the following statements are equivalent:

i) whenever $x, y \in \Omega$ and the segment joining $x$ and $y$ is also in $\Omega$ we have

$$
u(\lambda x+(1-\lambda) y) \leq \lambda u(x)+(1-\lambda) u(y)
$$

for all $0 \leq \lambda \leq 1$.

ii) $u$ is a viscosity subsolution of all equations

$$
F\left(x, u(x), D u(x), D^{2} u(x)\right)=0,
$$

where $F(x, z, p, M)$ is a continuous function in $\Omega \times \mathbb{R} \times \mathbb{R}^{n} \times \mathcal{S}^{n}$ satisfying (2.1). 
iii) $u$ is a viscosity subsolution of all linear equations with constant coefficients

$$
F\left(x, u, D u, D^{2} u\right)=-\operatorname{trace}\left(A \cdot D^{2} u\right)=0,
$$

where $A \in \mathcal{S}^{n}$ is positive definite.

iv) $u$ satisfies the inequality

$$
D^{2} u \geq 0
$$

in the viscosity sense.

When one of the above statements hold, the function is said to be convex.

Proof. The equivalence among Definitions ii), iii) and iv) follows by a linear algebra observation: A symmetric matrix $M$ is nonnegative definite (denoted by $M \geq 0$ ) if and only for all positive definite symmetric matrices A the inequality

$$
\operatorname{trace}(A \cdot M) \geq 0
$$

holds. The equivalence between i) and iv) is part of viscosity folklore. See [LMS] and [ALL].

We note that, in particular, convex functions are viscosity subsolutions of the following equations:

i) the Laplacian

$$
-\Delta u=0,
$$

ii) the $Q$-Laplacian for $Q>2$,

$$
-\operatorname{div}\left(|D u|^{Q-2} D u\right)=0,
$$

and

iii) the $\infty$-Laplacian

$$
-\sum_{i, j=1}^{n} \frac{\partial u}{\partial x_{i}} \frac{\partial u}{\partial x_{j}} \frac{\partial^{2} u}{\partial x_{i} \partial x_{j}}=0 .
$$

These are all equations that can be expressed as

$$
F\left(x, u(x), D u(x), D^{2} u(x)\right)=0
$$

for a continuous (this is the reason for $Q>2$ ) function $F$ satisfying (2.1).

Corollary 2.1. Let $u$ be convex in an open set $\Omega \subset \mathbb{R}^{n}$ and let $B_{R}$ be a ball such that $B_{4 R} \subset \Omega$. Then $u$ is locally bounded and we have the bound

$$
\|u\|_{L^{\infty}\left(B_{R}\right)} \leq C f_{B_{4 R}}|u| d x .
$$

Proof. A convex function $u$ is a viscosity subsolution of the Laplace equation. It then follows easily from the arguments in [CIL] that $u$ is subharmonic; that is, it satisfies the comparison principle with respect to harmonic functions. For a detailed argument, valid also for the $Q$-Laplacian, see [JLM]. Bounded sub-harmonic functions are in $W_{\text {loc }}^{1,2}$ and are weak subsolutions of the Laplace equation. See for example $[\mathrm{HKM}]$. Inequality (2.3) then follows easily for non-negative convex functions from the properties of weak subsolutions of the Laplace equation. For general convex functions a further argument with comparison with cones is needed. We present the details in the subelliptic case in the proof of the estimate (3.2) below. For a more classical proof see chapter 6 in [EG]. 
Corollary 2.2. Let $u$ be convex in an open set $\Omega \subset \mathbb{R}^{n}$ and let $B_{R}$ be a ball such that $B_{2 R} \subset \Omega$. Then $u$ is locally Lipschitz and we have the bound

$$
\|D u\|_{L^{\infty}\left(B_{R}\right)} \leq \frac{C}{R}\|u\|_{L^{\infty}\left(B_{2 R}\right)}
$$

Proof. A convex function is a viscosity subsolution of the $\infty$-Laplace equation. Jensen proved in [J2] that these subsolutions are Lipschitz and essentially obtained the inequality (2.4). A different proof can be found in [LM2].

\section{Convexity in the Viscosity Sense}

We choose the analogue of Definition iv) as our starting point. As in the previous section, upper-semicontinous functions are assumed to be not identically $-\infty$.

Definition 3.1. Let $\Omega \subset \mathcal{H}$ be an open set and $u: \Omega \rightarrow \mathbb{R}$ be an upper-semicontinuous function. We say that $u$ is convex in the viscosity sense, or just $v$-convex, in $\Omega$ if

$$
\left(D_{\mathrm{h}}^{2} u\right)^{*} \geq 0
$$

in the viscosity sense. That is, if $p \in \Omega$ and $\phi \in C^{2}$ touches $u$ from above at $p$ $(\phi(p)=u(p)$ and $\phi(q) \geq u(q)$ for $q$ near $p)$ we have $\left(D_{\mathrm{h}}^{2} \phi\right)^{*}(p) \geq 0$.

This definition is compatible with the Heisenberg group structure since v-convexity is preserved by left-translations and by dilations. Uniform limits of v-convex functions are v-convex and the supremum of a family of $\mathrm{v}$-convex functions is $\mathrm{v}$-convex since these results hold for viscosity subsolutions.

Similarly, we may consider the analogue to Definition iii).

Definition 3.2. Let $\Omega \subset \mathcal{H}$ be an open set and $u: \Omega \rightarrow \mathbb{R}$ be an upper-semicontinuous function. The function $u$ is v-convex if it is a viscosity subsolution of all linear equations with constant coefficients

$$
-\operatorname{trace}\left(A \cdot\left(D_{\mathrm{h}}^{2} u\right)^{\star}\right)=0,
$$

where $A \in \mathcal{S}^{2}$ is positive definite.

As in the Euclidean case these two Definitions 3.1 and 3.2 are equivalent. There is also an equivalent analogue to Definition ii), see Definition 3.3 below, involving fully nonlinear equations. The equations in question are of the form

$$
F\left(p, u(p), D u(p),\left(D_{\mathrm{h}}^{2} u\right)^{\star}(p)\right)=0
$$

where

$$
D u=\left(X_{1} u\right) X_{1}+\left(X_{2} u\right) X_{2}+\left(X_{3} u\right) X_{3}
$$

is the (complete) gradient of $u$.

A point of clarification is due here. From the non-isotropic point of view, the derivative $X_{3} u$ behaves like a second derivative since it belongs second term in the derived series $\mathfrak{h}_{2}=\left[\mathfrak{h}_{1}, \mathfrak{h}_{1}\right]$ of the Lie algebra $\mathfrak{h}$. However, it also behaves like a first derivative. Suppose $u: \Omega \rightarrow \mathbb{R}$ is a smooth function with an local maximum or minimum at $x_{0} \in \Omega$. Then, a simple calculation based on the stratified Taylor expansion gives

$$
X_{1} u\left(x_{0}\right)=0, X_{2} u\left(x_{0}\right)=0, \text { and } X_{3} u\left(x_{0}\right)=0 .
$$

This is the starting point of the theory of subelliptic jets developed in [B] and [MSt].

Consider continuous functions

$$
F: \mathcal{H} \times \mathbb{R} \times \mathbb{R}^{3} \times \mathcal{S}^{2} \rightarrow \mathbb{R}
$$


that are homogeneous, proper, and degenerate elliptic:

$$
\left\{\begin{aligned}
F(x, z, p, 0) & =0, \\
F(x, z, p, M) & \leq F\left(x, z^{\prime}, p, M\right) \text { if } z \leq z^{\prime}, \text { and } \\
F(x, z, p, M) & \leq F\left(x, z, p, M^{\prime}\right) \text { if } M^{\prime} \leq M
\end{aligned}\right.
$$

Definition 3.3. Let $\Omega \subset \mathbb{R}^{n}$ be an open set and $u: \Omega \rightarrow \mathbb{R}$ be an upper-semicontinuous function. The function $u$ is v-convex if it is a viscosity subsolution of all equations

$$
F\left(p, u(p), D u(p),\left(D_{\mathrm{h}}^{2} u\right)^{\star}(p)\right)=0,
$$

where $F(x, z, p, M)$ is a continuous functions satisfying (3.1).

This definition is equivalent to Definitions 3.1 and 3.2. It implies 3.1 since we can always take $F(x, z, p, M)=-\operatorname{trace}(A \cdot M)$ for positive definite $A \in \mathcal{S}^{2}$. It is implied by definition 3.2 since

$$
F\left(p, \phi(p), D \phi(p),\left(D_{\mathrm{h}}^{2} \phi\right)^{\star}\left(p_{0}\right)\right) \leq F(p, \phi(p), D \phi(p), 0)=0
$$

whenever $\phi \in C^{2}$ touches $u$ from above at $p_{0}$.

What about the analogue to Definition i)? This is the notion of h-convexity that we study in section $\S 4$ below.

We note that, in particular, convex functions are viscosity subsolutions of the following equations:

i) the Hörmander or Kohn Laplacian

$$
-\Delta_{\mathrm{h}} u=-\left(X_{1}^{2} u+X_{2}^{2}\right) u=0,
$$

ii) the subelliptic $Q$-Laplacian for $Q>2$,

$$
-\Delta_{Q, \mathrm{~h}} u=-\operatorname{div}_{\mathrm{h}}\left(\left|D_{\mathrm{h}} u\right|^{Q-2} D_{\mathrm{h}} u\right)=0,
$$

and

iii) the subelliptic $\infty$-Laplacian

$$
-\Delta_{\infty, \mathrm{h}} u=-\sum_{i, j=1}^{2}\left(X_{i} u\right)\left(X_{j} u\right)\left(X_{i} X_{j} u\right)=0 .
$$

Here $\operatorname{div}_{\mathrm{h}}$ is the natural divergence associated to the family $\left\{X_{1}, X_{2}\right\}$ and given by

$$
\operatorname{div}_{\mathrm{h}}\left(a X_{1}+b X_{2}\right)=-X_{1} a-X_{2} b \text {. }
$$

These are all equations that can be expressed as

$$
F\left(p, u(p), D u(p),\left(D_{\mathrm{h}}^{2} u\right)^{\star}(p)\right)=0
$$

for a continuous function $F$ satisfying (3.1). Next, we state the main theorem of this section:

Theorem 3.1. Let $\Omega \subset \mathcal{H}$ be an open set and $u: \Omega \rightarrow \mathbb{R}$ be a v-convex function. Let $B_{R}$ be a ball such that $B_{4 R} \subset \Omega$. Then $u$ is locally bounded and we have.

$$
\|u\|_{L^{\infty}\left(B_{R}\right)} \leq C f_{B_{4 R}}|u| d x .
$$

Moreover, $u$ is locally Lipschitz and we have the bound

$$
\left\|D_{\mathrm{h}} u\right\|_{L^{\infty}\left(B_{R}\right)} \leq \frac{C}{R}\|u\|_{L^{\infty}\left(B_{2 R}\right)} .
$$


Here $C$ is a constant independent of $u$ and $R$. If, in addition, $u$ is $C^{2}$, then the symmetrized horizontal second derivatives are nonnegative

$$
\left(D_{\mathrm{h}}^{2} u\right)^{*} \geq 0 \text {. }
$$

The general scheme of the proof will parallel the arguments of section $\S 2$ once we find a substitute for Euclidean cones. We will use the fact that v-convex functions are viscosity subsolutions of the Hörmander-Kohn Laplacian and viscosity subsolutions of the subelliptic $\infty$-Laplacian.

Let us first dispose of (3.4). This follows right away from the definition of vconvexity using $u$ as a test function.

To prove (3.2) and (3.3) we need to introduce a subelliptic analogue to a cone.

Definition 3.4. A cone based on the Carnot-Carathéodory ball $B_{R}(p)$ of radius $R$, centered at $p$ is the unique $\infty$-harmonic function $\omega_{R, p}$ in $B_{R}(p) \backslash\{p\}$ satisfying $\omega_{R, p}(p)=0$ and $\omega_{R, p}(q)=R$ for $q \in \partial B_{R}(p)$.

Note that for $Q>4$ a point has positive $Q$-capacity. Therefore, the Dirichlet problem in the punctured ball is well posed. The existence and uniqueness of cones as viscosity solutions of $-\Delta_{\infty, \mathrm{h}} u=0$ follows from [B]. A upper bound on cones follows easily from Theorem 1.1 .

Proposition 3.1. For $R>0$ and $p \in \mathcal{H}$ consider the cone $\omega_{R, p}$. We have the upper bound

for $q \in B_{R}(p)$.

$$
\omega_{R, p}(q) \leq d_{C C}(p, q)
$$

Proof. Write

$$
\omega_{R, p}(q)=\omega_{R, p}(q)-\omega_{R, p}(p) \leq\left\|D_{\mathrm{h}} \omega_{R, p}\right\|_{\infty} d_{C C}(p, q) .
$$

We need to check that $\left\|D_{\mathrm{h}} \omega_{R, p}\right\|_{\infty} \leq 1$. By Bieske's uniqueness Theorem, viscosity solutions of the subelliptic $\infty$-Laplacian can be expressed as the limit as $Q \rightarrow \infty$ of a sequence of $Q$-harmonic functions with the same boundary values. These $Q$-harmonic functions minimize $\left\|D_{\mathrm{h}} u\right\|_{Q}$ which easily implies that their limit minimizes $\left\|D_{\mathrm{h}} u\right\|_{\infty}$. That is, viscosity solutions of the subelliptic $\infty$-Laplacian are absolute minimizers of the the functional $\left\|D_{\mathrm{h}} u\right\|_{\infty}$ (see the proof of Theorem 3.2 below). Therefore we have

$$
\left\|D_{\mathrm{h}} \omega_{R, p}\right\|_{\infty} \leq\left\|D_{\mathrm{h}} d\right\|_{\infty}=1
$$

by Theorem 1.1 .

A lower bound follows from the Harnack inequality. We present a subelliptic version of the Harnack inequality for $\infty$-harmonic functions proved in the Euclidean case by Evans $[\mathrm{E}]$ for smooth functions and [LM] for viscosity solutions.

Theorem 3.2. Suppose $u$ is nonnegative viscosity solution of $-\Delta_{\infty, \mathrm{h}} u=0$ in a domain $\Omega$. Then, for all $\zeta \in C_{0}^{\infty}(\Omega)$ we have the inequality

$$
\left\|\zeta D_{\mathrm{h}} \log u\right\|_{\infty} \leq\left\|D_{\mathrm{h}} \zeta\right\|_{\infty} .
$$

Proof. The proof of this inequality parallels to the one of the Euclidean case given in $[\mathrm{LM}]$ once we have Bieske's extension [B] of Jensen's uniqueness [J2] result for viscosity solutions of $-\Delta_{\infty, \mathrm{h}} u=0$. For the reader's convenience, we include a complete proof in the subelliptic setting. 
One way to get estimates for $\infty$-harmonic functions, is to obtain estimates for $Q$-harmonic functions that remain stable as $Q \rightarrow \infty$. For the classical Moser-Serrin inequality, the constants blow up when $Q$ goes to $\infty$. An alternative method is to look for energy bounds for $D_{h} \log u$, for $Q$ large.

First, if $u_{Q} \geq 0$ is a nonnegative weak solution of the $Q$-Laplace equation

$$
\triangle_{Q} u=\operatorname{div}_{\mathrm{h}}\left(\left|D_{\mathrm{h}} u\right|^{Q-2} D_{\mathrm{h}} u\right)=0
$$

we have the inequality

$$
\left\|\zeta D_{\mathrm{h}} \log u_{Q}\right\|_{Q}^{Q} \leq\left(\frac{Q}{Q-1}\right)^{Q}\left\|D_{\mathrm{h}} \zeta\right\|_{Q}^{Q}
$$

To obtain inequality (3.6), use as a test function

$$
\frac{\zeta^{p}}{\varepsilon+u^{p-1}}
$$

in (3.5) and let $\varepsilon>0$ go to zero.

Select a bounded domain $D$ such that $\operatorname{supp} \zeta \subset D \subset \bar{D} \subset \Omega$. From a Theorem of $[\mathrm{B}]$ we have that $u$ is in $W^{1, \infty}(\Omega)$. For $Q>4$ consider the auxiliary problem:

$$
\left\{\begin{aligned}
\operatorname{div}\left(\left|D_{\mathrm{h}} u_{Q}\right|^{Q-2} D_{\mathrm{h}} u_{Q}\right) & =0 \\
u_{Q}-u & \in W_{0}^{1, Q}(D)
\end{aligned} \quad \text { in } D\right.
$$

Let $Q>4$. Then by the Hölder embedding Theorem (see [L1]) we have

$$
\left|u_{Q}(x)-u_{Q}(y)\right| \leq C d_{C C}(x, y)^{1-\frac{4}{Q}}|| D_{\mathrm{h}} u_{Q} \|_{L^{Q}(D)}
$$

for some constant $C$ independent of $u_{Q}$. Since $Q$-harmonic functions minimize the $L^{Q}$-norm of the gradient

$$
\left\|D_{\mathrm{h}} u_{Q}\right\|_{L^{Q}(D)} \leq\left\|D_{\mathrm{h}} u\right\|_{L^{Q}(D)} .
$$

It then follows that

$\left|u_{Q}(x)-u_{Q}(y)\right| \leq C d_{C C}(x, y)^{1-\frac{4}{Q}}|| D_{\mathrm{h}} u\left\|_{L^{Q}(D)} \leq C d_{C C}(x, y)^{1-\frac{4}{Q}}|D|^{\frac{1}{Q}}\right\| D_{\mathrm{h}} \|_{L^{\infty}(D)}$.

Let $u_{\infty}=\lim _{Q \rightarrow \infty} u_{Q}$, then

$$
\left|u_{\infty}(x)-u_{\infty}(y)\right| \leq c d(x, y)|| D_{h} u \|_{L^{\infty}(D)} .
$$

This shows that $u_{\infty}$ is locally Lipschitz in $\Omega$.

Given any positive integer $m$, we have for any $Q>m$

$$
\left(\frac{1}{|D|} \int_{D}\left|D_{h} u_{Q}\right|^{m}\right)^{\frac{1}{m}} \leq\left(\frac{1}{|D|} \int_{D}\left|D_{h} u_{Q}\right|^{Q}\right)^{\frac{1}{Q}} \leq\left(\frac{1}{|D|} \int_{D}\left|D_{h} u\right|^{Q}\right)^{\frac{1}{Q}} \leq\left\|D_{h} u\right\|_{L^{\infty}, D}
$$

Letting $Q \rightarrow \infty$ we get

$$
\left(\frac{1}{|D|} \int_{D}\left|D_{h} u_{\infty}\right|^{m}\right)^{\frac{1}{m}} \leq\left\|D_{h} u\right\|_{L^{\infty}, D}
$$

Next, letting $m \rightarrow \infty$, we derive

$$
\left\|D_{h} u_{\infty}\right\|_{L^{\infty}, D} \leq\left\|D_{h} u\right\|_{L^{\infty}, D}
$$

By the uniqueness result of $L^{\infty}$ minimizer in the subelliptic setting (see [B]), we conclude $u_{\infty}=u$.

Therefore, the Theorem follows by taking limit $Q \rightarrow \infty$ in the inequality for $u_{Q}$. 
Given $B_{r}^{\mathcal{H}}$ and $B_{R}^{\mathcal{H}}$ two concentric balls with respect to the smooth Heisenberg gauge in $\Omega$ with radius $r$ and $R$. We can always find a smooth function $\zeta$, a radial function of the smooth gauge, such that $\zeta=1$ on $B_{r}^{\mathcal{H}}$ and 0 outside $B_{R}^{\mathcal{H}}$, and $\left|D_{h} \zeta\right| \leq \frac{C}{R-r}$. From the above Theorem we obtain

$$
\left\|D_{\mathrm{h}} \log u\right\|_{B_{r}^{\mathcal{H}}, \infty} \leq \frac{C}{R-r} .
$$

This easily implies the following:

Corollary 3.1. Let $u$ be a non-negative viscosity solution of $-\Delta_{\infty, \mathrm{h}} u=0$ in a domain $\Omega$. Let $B$ be a ball with respect to the smooth gauge such that $2 B \subset \Omega$. Then, there exists a constant $C$ so that

$$
\sup _{B} u \leq C \inf _{B} u
$$

In fact it follows from (3.7) that

$$
u(p) \leq \exp \left\{C\left(\frac{d_{H}(p, q)}{R-r}\right)\right\} u(q) .
$$

A consequence of the Harnack inequality that we mention in passing is the following Liouville Theorem:

Theorem 3.3. The only $\infty$-harmonic functions bounded from below in the whole group $\mathcal{H}$ are the constants.

We now return to the subelliptic cones.

Proposition 3.2. The cone $\omega_{R, p}$ satisfies

$$
\inf \left\{\omega_{R, p}(q): \frac{R}{2} \leq d_{C C}(p, q) \leq R\right\} \geq C_{0} R,
$$

where $C_{0}$ is an absolute constant independent of $R$ and $p$.

Proof. By dilation and translation invariance, we may assume that $p=0$ and $R=1$. From the Harnack inequality (Corollary 3.1) we deduce

$$
\inf \left\{\omega_{1,0}(q): d_{C C}(0, q)=\frac{1}{2}\right\}=C_{0}>0 \text {. }
$$

The lemma follows then from the comparison principle.

Lemma 3.1. Let $u$ be v-convex, then $u$ is locally bounded.

Proof. Without loss of generality, we may assume that $u$ is locally bounded from above because $u$ is upper semicontinuous. We may further assume that $u \leq 0$. Suppose $u(p)=-\infty, p \in \Omega$ and $B(p, R) \subset \Omega$. Then for $k>0$ we have

$$
u(q) \leq-k\left(R-\omega_{R, p}(q)\right)
$$

for $q \in \partial B(p, R) \cup\{p\}$. From Bieske's comparison principle we conclude that this inequality is indeed valid for $q \in \partial B(p, R)$. Let $k \rightarrow \infty$ to get $u(q)=-\infty$ on $B(p, R)$. Consequently $u(q)=-\infty$ in $\Omega$ by covering $\Omega$ with chains of balls. This is a contradiction. 
Suppose now that for some $p \in \Omega$ there is a sequence $p_{k} \rightarrow p$ satisfying $u\left(p_{k}\right) \leq$ $-k R$. Then we can conclude in the same way as above that

$$
u(q) \leq-k\left(R-\omega_{R, p_{k}}(q)\right)
$$

for $q \in B\left(p_{k}, R\right)$. In particular we must have

$$
u(p) \leq-k\left(R-\omega_{R, p_{k}}(p)\right) .
$$

Using the fact $\omega_{R, p_{k}}(p) \leq d_{C C}\left(p_{k}, q\right)$ for $k$ large enough we have

$$
u(p) \leq-k \frac{r}{2}
$$

Letting $k \rightarrow \infty$, we conclude that $u(p)=-\infty$. This is again a contradiction.

Lemma 3.2. A viscosity subsolution of the Hörmander-Kohn Laplacian $-\Delta_{\mathrm{h}}$ is subharmonic.

Proof. This is standard and we include it for the sake of completeness. Let $u$ be a viscosity subsolution. Suppose that we have a bounded domain $\Omega \subset \mathcal{H}$ and a function $h$ in $\Omega$ such that

$$
-\Delta_{\mathrm{h}} h_{\epsilon}=\epsilon>0
$$

and

$$
\limsup _{q \rightarrow p} u(q) \leq \liminf _{q \rightarrow p} h_{\epsilon}(q)
$$

where $q \in \Omega$, for all $p \in \partial \Omega$ and both sides are not simultaneously $+\infty$ or $-\infty$. We claim that $u(q) \leq h_{\epsilon}(q)$ for all $q \in \Omega$.

If this is not the case $u-h_{\epsilon}$ has a positive interior maximum, say at the point $q_{0} \in \Omega$. Let $m=v\left(q_{0}\right)-h_{\epsilon}\left(q_{0}\right)>0$. Consider the smooth function $h_{\epsilon}+m$. It touches $u$ from above at the point $q_{0}$. By the definition of viscosity subsolution we get $-\Delta_{\mathrm{h}} h_{\epsilon}\left(q_{0}\right) \leq 0$ in contradiction with (3.8).

The case where $\epsilon=0$ follows by approximating $h$ by $h_{\epsilon}=h-\frac{\epsilon}{2}\left(x^{2}+y^{2}\right)$ and noticing that if $u-h$ has a positive interior local minima, so must be $u-h_{\epsilon}$ for small $\epsilon>0$.

Lemma 3.3. Bounded sub-harmonic functions are weak subsolutions of the Hörmander-Kohn Laplacian.

The point here is to show that bounded sub-harmonic functions are in $W^{1,2}$. This is certainly known, but we have been unable to locate a reference.

Proof. Once again we need to adapt a well-known argument in the Euclidean case to the case of the Heisenberg group. See Chapters 3 and 7 of [HKM] for the degenerate quasiliner case in $\mathbb{R}^{n}$. We need the fact that balls with respect to the smooth Heisenberg gauge are regular sets for the Dirichlet problem for the Hörmander-Kohn Laplacian (for example see [Ga] and [LU]).

Let $u$ be a bounded sub-harmonic function in a domain $\Omega$. Select a regular subdomain $D$ whose closure is $\bar{D} \subset \Omega$. Choose a decreasing sequence of functions $\phi_{i} \in C^{\infty}$ converging to $u$ in $\bar{D}$. Let $u_{i}$ be the solution to the obstacle problem in $D$ with obstacle $\phi_{i}$. The existence of this solution follow from a variational principle as in the Euclidean case. That is, the function $u_{i} \in C(\bar{D}) \cap W^{1,2}(D)$ and $u_{i}=\phi_{i}$ on $\partial D$, each $u_{i}$ is subharmonic in $D$, and harmonic in the open set 
$U_{i}=\left\{x \in D: u_{i}<\phi_{i}\right\}$. The sequence $u_{i}$ is decreasing and $u \leq u_{i}$ in $U_{i}$ by the comparison principle since

$$
\limsup _{x \rightarrow y} u(x) \leq u(y) \leq \phi_{i}(y)=\lim _{x \rightarrow y} u_{i}(x)
$$

for all $y \in \partial U_{i}$. Conclude that $u \leq u_{i}$ in $D$ and

$$
u=\lim _{i \rightarrow \infty} \phi_{i} \geq \lim _{i \rightarrow \infty} u_{i} \geq u
$$

in $D$.

We have shown the existence of a decreasing sequence of subsolutions $u_{i} \in$ $C(\bar{D}) \cap W^{1,2}(D)$ such that $u=\lim _{i \rightarrow \infty} u_{i}$ in $D$. Moreover, the functions $u_{i}$ are subharmonic in $D$. If, in addition, $u$ is bounded, we have a uniform bound for $u_{i}$. It then follows by Caccioppoli's inequality [L2] that the sequence $u_{i}$ is, in fact, uniformly bounded in $W^{1,2}\left(D^{\prime}\right)$ for any subdomain $D^{\prime}$ compactly contained in $D$. It then follows by standard limit theorems that $u \in W_{\text {loc }}^{1,2}$ and $u$ is a weak subsolution.

Estimate (3.2) for non-negative convex functions follows by combining Lemmas $3.1,3.2$, and 3.3 with well known properties of nonnegative weak subsolutions. See for example [L2]. In fact since $u^{+}$is convex, this shows that for $u^{+}$we always have

$$
\left\|u^{+}\right\|_{L^{\infty}\left(B_{R}\right)} \leq C f_{B_{2 R}} u^{+} d x .
$$

To present an argument valid for general $u$ we need the fact that $u$ is continuous. This follows from Proposition 3.3 below, whose proof is independent of (3.2). Suppose then that $u$ is continuous and bounded as we may. Consider a ball $B_{R}$ such that $B_{2 R} \subset \Omega$. If we denote by

$$
M_{2 R}^{+}=\left\|u^{+}\right\|_{L^{\infty}\left(B_{2 R}\right)}
$$

then $v=u-M_{2 R}^{+}$is a negative convex function in $B_{R}$. Fix a point $p \in B_{R}$, set $S=d_{C C}\left(p, \partial B_{2 R}\right) \geq R$ and consider the inequality

$$
v(q) \leq \frac{v(p)}{S}\left(S-\omega_{S, p}(q)\right)
$$

for $q \in B_{S}(p)$, where the cone $\omega_{S, p}$ is the cone based on the ball $B_{S}(p)$. Inequality (3.10) holds at the center $q=p$ and at the boundary $q \in \partial B_{S}(p)$ since $v$ is negative. By the comparison principle we conclude that (3.10) holds for all $q \in B_{R}(p)$. Using Proposition 3.2 we conclude

$$
v(q) \leq C_{1} v(p)
$$

whenever $d_{C C}(p, q) \leq S / 2$. For $q \in B_{S / 2}(p)$ we then have

$$
u(q)-M_{2 R}^{+} \leq C_{1}\left(u(p)-M_{2 R}^{+}\right) .
$$

Choose $p \in B_{R / 2}$ to be a point for which

$$
\left\|u^{-}\right\|_{L^{\infty}\left(B_{R}\right)}=-u^{-}(p),
$$

which in turn gives

$$
u(q)-M_{2 R}^{+} \leq C_{1}\left(-\left\|u^{-}\right\|_{L^{\infty}\left(B_{R}\right)}-M_{2 R}^{+}\right)
$$

Therefore, for all $q \in B_{R / 2}(p)$ we obtain

$$
C_{1}\left\|u^{-}\right\|_{L^{\infty}\left(B_{R}\right)} \leq-u(q)+\left(C_{1}+1\right) M_{2 R}^{+} .
$$


Averaging on the ball $B_{R / 2}(p)$ and using (3.9) we get (3.2).

Proposition 3.3. If $u$ is a viscosity subsolution of the subelliptic $\infty$-Laplacian in a domain $\Omega \subset \mathcal{H}$, then $u$ is locally Lipschitz. Moreover, we have the bound

$$
\left|D_{\mathrm{h}} u(p)\right| \leq \frac{C\|u\|_{\Omega, \infty}}{\operatorname{dist}_{C C}(p, \partial \Omega)},
$$

for a. e. $p \in \Omega$ and $C$ is an constant independent of $u$.

Proof. This is a subelliptic version of Jensen's original proof [J2] where the Euclidean cones are replaced by the cones introduced in definition 3.4. Denote by $M=\|u\|_{\Omega, \infty}$ and for $\epsilon>0$ consider the subdomain

$$
\Omega_{\epsilon}=\left\{p \in \Omega: d_{C C}(p, \partial \Omega)>\epsilon\right\} .
$$

We want to estimate the supremum

$$
\sup _{p, q \in \Omega_{\epsilon}} \frac{|u(p)-u(q)|}{d_{C C}(p, q)} .
$$

Fix two poinst $p_{0}, q_{0} \in \mathcal{H}$. If $d_{C C}\left(p_{0}, q_{0}\right) \geq \epsilon$, we clearly have

$$
\frac{\left|u\left(p_{0}\right)-u\left(q_{0}\right)\right|}{d\left(p_{0}, q_{0}\right)} \leq \frac{2 M}{\epsilon}=\lambda .
$$

Suppose now that $d_{C C}\left(p_{0}, q_{0}\right)<\epsilon$. We will argue by using the comparison with the cones $\omega_{\epsilon, p_{0}}$ and $\omega_{\epsilon, q_{0}}$.

For $p \in \partial B\left(p_{0}, \epsilon\right) \cup\left\{p_{0}\right\}$, we have

$$
u(p)-u\left(p_{0}\right) \leq \lambda \omega_{\epsilon, p_{0}}(p) .
$$

and for $q \in \partial B\left(q_{0}, \epsilon\right) \cup\left\{q_{0}\right\}$, we have

$$
u(q)-u\left(q_{0}\right) \leq \lambda \omega_{\epsilon, q_{0}}(q) .
$$

By Bieske's comparison principle, the above inequalities hold inside their respective balls. Since $d_{C C}\left(p_{0}, q_{0}\right)<\epsilon$, we conclude

$$
u\left(q_{0}\right)-u\left(p_{0}\right) \leq \lambda \omega_{\epsilon, p_{0}}\left(q_{0}\right)
$$

and

$$
u\left(p_{0}\right)-u\left(q_{0}\right) \leq \lambda \omega_{\epsilon, q_{0}}\left(p_{0}\right) .
$$

It follow from Lemma 3.1 that $\omega_{\epsilon, p_{0}}\left(q_{0}\right) \leq d_{C C}\left(p_{0}, q_{0}\right)$ and $\omega_{\epsilon, q_{0}}\left(p_{0}\right) \leq d_{C C}\left(p_{0}, q_{0}\right)$ and these two inequalities imply

$$
\left|u\left(p_{0}\right)-u\left(q_{0}\right)\right| \leq \lambda d\left(p_{0}, q_{0}\right)
$$

Combining Lemma 3.1 and Lemma 3.3, we have completed the proof of inequality 3.3, and therefore, that of Theorem 3.1. 


\section{Horizontal ConveXity}

Given a point $p \in \mathcal{H}$ the line segment joining $p \cdot h^{-1}$ to $p \cdot h$, denoted by $\left[p \cdot h^{-1}, p \cdot h\right]$ is always contained in the horizontal subspace $p \cdot \mathcal{H}_{0}$.

Definition 4.1. Let $\Omega$ be an open set in $\mathcal{H}$ and $u: \Omega \rightarrow \mathbb{R}$ be an upper-semicontinuous function. We say that $u$ is horizontally convex or just $h$-convex in $\Omega$, if for all $p \in \Omega$ and $h \in \mathcal{H}_{0}$ such that $\left[p \cdot h^{-1}, p \cdot h\right] \subset \Omega$ we have

$$
u(p) \leq \frac{1}{2}\left\{u(p \cdot h)+u\left(p \cdot h^{-1}\right)\right\} .
$$

Remark 1. If in this definition we require the point $h$ to be in $\mathcal{H}$ rather than $\mathcal{H}_{0}$ we obtain a notion that we could call group convexity. This definition is clearly stronger than h-convexity. It coincides with the Euclidean convexity with respect to the coordinates $(x, y, z)$. This is the case because we always have

$$
\frac{p \cdot h+p \cdot h^{-1}}{2}=p .
$$

By using the Campbell-Haussdorff formula, one can easily see that for any Carnot group of step 2, group convexity is just Euclidean convexity.

Let us consider a point $p$ in $\mathcal{H}$ and a point $q \in T_{h}(p)$. We can write $q=p \cdot h$, for some horizontal $h \in \mathcal{H}_{0}$. The segment $[p, q]$ joining $p$ and $q$ on $T_{h}(p)$ is also contained in $T_{h}(q)$. In fact, writing $p_{t}=p \cdot \delta_{t}(h)$ for a point in this segment, we need to check that $p_{t}=q \cdot h^{\prime}$ for some $h^{\prime} \in \mathcal{H}_{0}$. The choice $h^{\prime}=h \cdot \delta_{t}(h)$ works. Taking into account this remark, it is now natural to consider the following definition due to Cabré and Caffarelli [Ca].

Definition 4.2. An upper semi-continuous function u is $C C$-convex in $\Omega$ if for any two points $p$ and $q \in \Omega$ such that the segment $[p, q] \subset \Omega \cap p \cdot \mathcal{H}_{0}$, we have that the restriction of $u$ to $[p, q]$ is a convex function of the variable $t$ in the parametrization $p \cdot \delta_{t}(h)$ of the segment $[p, q]$.

The two definitions are indeed equivalent.

Lemma 4.1. An upper semi-continuous function is $h$-convex if and only if it is CC-convex.

Proof. Let us begin with assuming CC-convexity. If h-convexity fails, then there exist a point $p \in \Omega$ and a horizontal vector $h \in \mathcal{H}_{0}$ such that

$$
u(p)>\frac{u(p \cdot h)+u\left(p \cdot h^{-1}\right)}{2}
$$

Set $q=p \cdot h^{-1}$ and consider the function of one variable:

$$
U(t)=u\left(q \cdot \delta_{t}\left(h^{2}\right)\right)
$$

Rewriting the previous inequality in terms of $U(t)$, we get

$$
U\left(\frac{1}{2}\right)>\frac{U(0)+U(1)}{2}
$$

contradicting the CC-convexity property.

To prove the converse note that we assume that $u$ is continuous by using the fact that h-convex functions are v-convex and therefore Lipschitz continuous. See Proposition 4.2 below. Suppose there exist $p, q \in \Omega$ such that the segment $\Omega \cap p \cdot \mathcal{H}_{0}$ 
and the function $u$ restricted to the segment $[p, q]$ is not convex. Then, there exists points $p^{\prime}, q^{\prime} \in[p, q]$ such that

$$
\frac{u\left(p^{\prime}\right)+u\left(q^{\prime}\right)}{2}<u\left(\frac{p^{\prime}+q^{\prime}}{2}\right)
$$

in contradiction with h-convexity at the point $\left(p^{\prime}+q^{\prime}\right) / 2$

Both definitions of convexity are compatible with the Carnot group structure: they are left-invariant and dilation-invariant. Note also that the supremum of a family of h-convex functions and the uniform limit of a sequence of $\mathrm{h}$-convex functions are h-convex. An important property of h-convex functions is that they are always (locally) uniform limits of smooth h-convex functions. This follows easily by using using left group convolution with a mollifier (see proof of Theorem 4.2 below.)

A typical example of h-convex function which is not group convex is given by the function

$$
u(x, y, z)=\left(x^{2}+y^{2}\right)-\varepsilon z^{2}
$$

which is h-convex in a neighborhood of zero as it is clear from the subelliptic Taylor's formula ([FS], Chapter 1).

Lemma 4.2. Let $u$ be $h$-convex in $\Omega$ then $u$ is $v$-convex in $\Omega$.

Proof. Let $\phi$ be a test function touching $u$ from above at the point $p_{0} \in \Omega$. This means $u\left(p_{0}\right)=\phi\left(p_{0}\right)$ and $u(p) \leq \phi(p)$ for $p$ in a neighborhood of $p_{0}$.

Suppose that $\left(D_{\mathrm{h}}^{2} \phi\right)^{*}\left(p_{0}\right)$ is not positive definite. Let write the Taylor expansions as in $[\mathrm{B}]$ and $[\mathrm{M}]$ :

$$
\phi\left(p_{0} \cdot z\right)=\phi\left(p_{0}\right)+\left\langle D_{\mathrm{h}} \phi\left(p_{0}\right), z\right\rangle+\frac{1}{2}\left\langle\left(D_{\mathrm{h}}^{2} \phi\right)^{*}\left(p_{0}\right) z, z\right\rangle+o\left(|z|^{2}\right)
$$

and

$$
\phi\left(p_{0} \cdot z^{-1}\right)=\phi\left(p_{0}\right)-\left\langle D_{\mathrm{h}} \phi\left(p_{0}\right), z\right\rangle+\frac{1}{2}\left\langle\left(D_{\mathrm{h}}^{2} \phi\right)^{*}\left(p_{0}\right) z, z\right\rangle+o\left(|z|^{2}\right) .
$$

Adding these two equations and using the fact the $\phi$ touches $u$ from above at $p_{0}$ we get

$$
\frac{u\left(p_{0} \cdot z\right)+u\left(p_{0} \cdot z^{-1}\right)}{2} \leq u\left(p_{0}\right)+\frac{1}{2}\left\langle\left(D_{\mathrm{h}}^{2} \phi\right)^{*}\left(p_{0}\right) z, z\right\rangle+o\left(|z|^{2}\right) .
$$

For some $z$ near zero this implies

$$
\frac{u\left(p_{0} \cdot z\right)+u\left(p_{0} \cdot z^{-1}\right)}{2}<u\left(p_{0}\right)
$$

contradicting the h-convexity of $u$.

Lemma 4.3. If $u \in C^{2}(\Omega)$, then $u$ satisfies the following horizontal Taylor's formula with integral reminder:

$$
u(p \cdot z)=u(p)+\left\langle D_{\mathrm{h}} u(p), z\right\rangle+\int_{0}^{1}\left\langle\left(D_{\mathrm{h}}^{2} u\left(p \cdot \delta_{t}(z)\right)\right)^{*} z, z\right\rangle(1-t) d t
$$

where $z \in \mathcal{H}_{0}$. 
Proof. Let $\gamma(t)$ be the horizontal curve connecting $p$ and $p \cdot z$ such that $\gamma(0)=p$ and $\gamma(1)=p \cdot z$. Then

$$
\begin{aligned}
u(p \cdot z)-u(p) & =\quad \int_{0}^{1} \frac{d}{d t} \gamma(t) d t \\
& =\int_{0}^{1}<D_{\mathrm{h}} u(\gamma(t)), \gamma^{\prime}(t)>d t
\end{aligned}
$$

In particular, if we take $\gamma(t)=p \cdot \delta_{t} z$, then $\gamma^{\prime}(t)=z$. Therefore,

$$
u(p \cdot z)-u(p)=\int_{0}^{1}<D_{\mathrm{h}} u\left(p \cdot \delta_{t} z\right), z>d t .
$$

By using integration by parts, the right-hand side of the above inequality is equal to

$$
\begin{aligned}
& -\int_{0}^{1}<D_{\mathrm{h}} u\left(p \cdot \delta_{t} z\right), z>d(1-t) \\
= & -<D_{\mathrm{h}} u\left(p \cdot \delta_{t} z\right), z>\left.(1-t)\right|_{0} ^{1}+\int_{0}^{1}<\left(D_{\mathrm{h}}^{2}\right)^{*} u\left(p \cdot \delta_{t} z\right) z, z>(1-t) d t \\
= & <D_{\mathrm{h}} u(p), z>+\int_{0}^{1}<\left(D_{\mathrm{h}}^{2}\right)^{*} u\left(p \cdot \delta_{t} z\right) z, z>(1-t) d t
\end{aligned}
$$

Proposition 4.1. If $u \in C^{2}(\Omega)$ and $u$ is $v$-convex in $\Omega$, then $u$ is $h$-convex in $\Omega$.

Proof. By using $u$ as a test function in the definition of v-convexity it follows that $\left(D_{\mathrm{h}}^{2} u(p)\right)^{*} \geq 0$ in $\Omega$. Suppose now that $u$ fails to be h-convex at a point $p$. For some $z \in \mathcal{H}_{0}$ we then have

$$
\frac{u(p \cdot z)+u\left(p \cdot z^{-1}\right)}{2}<u(p)
$$

Without loss of generality we may assume that $p=0 \in \Omega$. By the horizontal Taylor expansion with integral reminder

$$
u(z)=u(0)+\left\langle D_{\mathrm{h}} u(0), z\right\rangle+\int_{0}^{1}\left\langle\left(D_{\mathrm{h}}^{2} u\left(\delta_{t}(z)\right)\right)^{*} z, z\right\rangle(1-t) d t
$$

and

$$
u\left(z^{-1}\right)=u(0)-\left\langle D_{\mathrm{h}} u(0), z\right\rangle+\int_{0}^{1}\left\langle\left(D_{\mathrm{h}}^{2} u\left(\delta_{t}\left(z^{-1}\right)\right)\right)^{*} z, z\right\rangle(1-t) d t .
$$

Adding these two formulas we obtain

$$
\int_{0}^{1}\left\langle\left(D_{\mathrm{h}}^{2} u\left(\delta_{t}\left(z^{-1}\right)\right)\right)^{*}+\left(D_{\mathrm{h}}^{2} u\left(\delta_{t}(z)\right)\right)^{*} z, z\right\rangle(1-t) d t<0 .
$$

Since the integrand is positive we have arrived to a contradiction.

Next, we consider the existence of second derivatives. In the Euclidean case, the classical Alexandrov's Theorem asserts that convex functions are a. e. pointwise twice differentiable. One way to prove this (see Chapter 6 in [EG]) is to see that convex functions have second distributional derivatives which are measures and to use some differentiation theorems in conjunction with Corollaries 2.1 and 2.2. 
Recently, Ambrosio and Magnani ([AM]) have proved a weak Aleksandrov-type differentiability theorem for functions on Carnot Groups whose horizontal distributional derivatives up to order 2 are measures. The space of such functions is denoted by $B V_{h}^{2}$. Namely, the statement of the theorem is:

Theorem 4.1. Let $u$ be a $B V_{h}^{2}(\Omega)$. For a. e. $p \in \Omega$ there exists a polynomial $\mathcal{P}_{[p]}$ with degree less or equal than 2 , such that

$$
\lim _{r \rightarrow 0^{+}} \frac{1}{r^{2}} f_{B(p, r)}\left|u(q)-\mathcal{P}_{[x]}(q)\right| d q=0 .
$$

However, for h-convex functions $u$ we can only prove that the symmetrized second order horizontal derivatives $\frac{X_{i} X_{j}+X_{j} X_{i}}{2} u$ are measures. We do not know if the nonsymmetric part $X_{3} u$ is always a Radon measure, and thus, we cannot yet answer the question whether always an h-convex function $u$ is in the space $B V_{h}^{2}$.

Theorem 4.2. If $u$ is a h-convex function in an open set $\Omega \subset \mathcal{H}$, then $\frac{X_{i} X_{j}+X_{j} X_{i}}{2} u$ is a Radon measure for $i, j=1,2$.

The proof is an adaption of the Euclidean proof based on the Riesz representation Theorem.

Proof. Let $\phi \in C_{0}^{\infty}$ such that $\operatorname{supp}(\phi) \subset B(0,2)$ and $\phi=1$ on $B(0,1)$. Define $\phi_{\epsilon}(p)=\epsilon^{-4} \phi\left(\delta_{\frac{1}{\epsilon}} p\right)$ for any $p \in \mathbb{H}$. Consider the mollification $u_{\epsilon}=\phi_{\epsilon} * u$ of $u$ defined by the group convolution

$$
\phi_{\epsilon} * u(q)=\int_{\mathcal{H}} u\left(y^{-1} \cdot q\right) \phi_{\epsilon}(y) d y
$$

where $q \in \Omega_{\epsilon}=\{q \in \Omega$ : $\operatorname{dist}(q, \partial \Omega)>2 \epsilon\}$. Then $u_{\epsilon}$ is a smooth h-convex function in $\Omega_{\epsilon}$, since for a horizontal $w \in \mathcal{H}_{0}$ such that the segment $\left[p w^{-1}, p w\right] \subset \Omega_{\epsilon}$ we have

$$
u_{\epsilon}(p w)+u_{\epsilon}\left(p w^{-1}\right)-2 u_{\epsilon}(p)=\int_{\mathcal{H}}\left[u\left(y^{-1} p w\right)+u\left(y^{-1} p w^{-1}\right)-2 u\left(y^{-1} p\right)\right] \phi_{\epsilon}(y) d y
$$

and the integrand is nonnegative. Since $u_{\epsilon}$ is smooth, we conclude $\left(D_{\mathrm{h}}^{2}\right) u_{\epsilon}(p) \geq 0$ for $p \in \Omega_{\epsilon}$. Given any constant vector $\xi=\left(\xi_{1}, \xi_{2}\right)$ define a linear functional on the space of functions $\Psi \in C_{0}^{\infty}\left(\Omega_{\epsilon}\right)$ by

$$
L_{\epsilon}(\Psi)=\sum_{i, j=1}^{2} \int_{\Omega_{\epsilon}} u_{\epsilon}(p)\left(X_{i} X_{j}\right) \Psi(p) \xi_{i} \xi_{j} d p .
$$

We can write this functional in the form

$$
L_{\epsilon}(\Psi)=\sum_{i, j=1}^{2} \int_{\Omega_{\epsilon}} u_{\epsilon}(p)\left(\frac{X_{i} X_{j}+X_{j} X_{i}}{2}\right) \Psi(p) \xi_{i} \xi_{j} d p .
$$

Integration by parts shows that

$$
L_{\epsilon}(\Psi)=\sum_{i, j=2} \int_{\Omega_{\epsilon}} \Psi(p)\left(\frac{X_{i} X_{j}+X_{j} X_{i}}{2}\right) u_{\epsilon}(p) \xi_{i} \xi_{j} d p .
$$

In view of the fact that $u_{\epsilon}$ is smooth and h-convex

$$
\sum_{i, j=1}^{2}\left(\frac{X_{i} X_{j}+X_{j} X_{i}}{2}\right) u_{\epsilon}(p) \xi_{i} \xi_{j} \geq 0
$$


for all $p \in \Omega_{\epsilon}$ and $\xi=\left(\xi_{1}, \xi_{2}\right) \in \mathbb{R}^{2}$. Therefore, we conclude that $L_{\epsilon}$ is a positive functional $L_{\epsilon}(\Psi) \geq 0$ for $\Psi \geq 0$. This is the key step where the symmetrization of the horizontal second derivatives is essential.

We now define

$$
L(\Psi)=\sum_{i, j=1}^{2} \int_{\Omega} u(p)\left(\frac{X_{i} X_{j}+X_{j} X_{i}}{2}\right) \Psi(p) \xi_{i} \xi_{j} d p
$$

for $\Psi \in C_{0}^{\infty}(\Omega)$. Then, since $u_{\epsilon} \rightarrow u$ locally uniformly in $\Omega$ we have

$$
L(\Psi)=\lim _{\epsilon \rightarrow 0} L_{\epsilon}(\Psi) \geq 0 .
$$

Hence, by the Riesz's representation Theorem, we find a measure $d \mu^{\xi}$ in $\Omega$ such that

$$
L(\Psi)=\int_{\Omega} \Psi d \mu^{\xi}
$$

Choose $\xi=e^{1}=(1,0)$ or $\xi=e^{2}=(0,1)$. We can write

$$
\int_{\Omega} u(p) X_{i}^{2} \Psi(p) d p=\int_{\Omega} \Psi d \mu^{i i}
$$

Next, we choose $\xi=\frac{e_{1}+e_{2}}{\sqrt{2}}$ to conclude the existence of a measure $d \mu^{i j}$ such that

$$
\int_{\Omega} u(p)\left(\frac{X_{i} X_{j}+X_{j} X_{i}+X_{i}^{2}+X_{j}^{2}}{2}\right) \Psi(p) d p=\int_{\Omega} \Psi d \mu^{i j} .
$$

Therefore, we can write

$$
\int_{\Omega} u(p)\left(\frac{X_{i} X_{j}+X_{j} X_{i}}{2}\right) \Psi(p) d p=\int_{\Omega} \Psi\left(d \mu^{i j}-d \mu^{i i}-d \mu^{j j}\right) .
$$

Thus, we have shown that in the sense of distributions

$$
\frac{X_{i} X_{j}+X_{j} X_{i}}{2} u=d \mu^{i j}-d \mu^{i i}-d \mu^{j j}
$$

Remark 2. The classes of h-convex, CC-convex and v-convex functions can also be studied in general Carnot groups. Lemmas 4.1, 4.2, 4.3, and Proposition 4.1 hold with the same proofs. Similarly, the given proof Theorem 4.2 can be easily generalized. However, our results on section $\S 3$ depend on the comparison principle for $\infty$-subharmonic and $\infty$-superharmonc functions which, at the moment, it has only being verified in the Heisenberg group ([B].)

Remark 3. Most of the results of this paper have been presented by the authors in several invited talks: B. Stroffolini, Bressanone (July 2000), Paris (October 2000), Trento (June 2001), Edinburgh (July 2001), Pisa and Padova (February 2002), J. Manfredi, Pacific Institute of Mathematics at Vancouver (July 2001), University of Bern (December 2001) and University of Maryland (May 2002) and G. Lu, AMS Meeting in Ann Arbor (March 2002) and Cornell University (April 2002). We would like to thank the organizers of these meetings/seminars for their invitations. 


\section{REFERENCES}

[AM] Ambrosio, L. and Magnani, V., Some fine properties of BV functions on Sub-Riemannian groups, preprint n.2, Scuola Normale Superiore, Pisa, Febbraio 2002.

[ALL] Alvarez, O., Lasry, J.M., and Lions, P.L., Convex viscosity solutions and state constraints, J. Math. Pures Appl. 9 (76), no. 3, 1997, 265-288.

[B] Bieske, T., On $\infty$-harmonic functions on the Heisenberg group, Comm. in Partial Differential Equations 27 ( 3 \& 4), 2002, 727-762.

[BR] Bellaïche, André and Risler, Jean-Jacques, ed., Sub-Riemannian Geometry, Progress in Mathematics, Vol. 144, Birkhäuser, 1996.

[Ca] Cabré, X., personal communication.

[C] Crandall, M., Viscosity Solutions: A Primer, Lecture Notes in Mathematics 1660, Springer-Verlag, 1997, pp. 1-43.

[CC1] Caffarelli, L. and Cabré, X. Fully nonlinear elliptic equations, volume 43 of AMS colloquium publications, AMS, Providence, RI, 1995.

[CEL] Crandall, M., Evans C. and Lions, P.-L., Some properties of viscosity solutions of Hamilton-Jacobi equations, Trans. of Amer. Math. Soc., Vol. 282(2), pp. 487-502, 1984.

[CIL] Crandall, M., Ishii, H., and Lions, P. L., User's Guide to Viscosity Solutions of Second Order Partial Differential Equations, Bull. of Amer. Math. Soc., Vol. 27, No. 1, July 1992, pp. 1-67.

[DGN] Danielli, D., Garofalo, N. and Nhieu, D., Notions of convexity in Carnot groups, preprint.

[E] Evans, L.C., Estimates for smooth absolutely minimizing Lipschitz extensions, Electronic Journal of Differential Equations 1993(3), 1-10.

[E2] Evans, L.C., Partial Differential Equations, Graduate Studies in Mathematics 19, American Mathematical Society, Providence, RI, 1998.

[EG] Evans, C. and Gariepy, R., Measure theory and fine properties of functions, Studies in Advanced Mathematics, CRC Press LLC, 1992.

[FS] Folland, G.B., and Stein, E. M., Hardy Spaces on Homogeneous Groups, Princeton University Press, Princeton, NJ, 1982.

[Ga] Gaveau, B., Principle de moindre action, propagation de la chaleur, et estimées souselliptiques sur certain groups nilpotents, Acta Mathematica 139(1977), no. 1-2, 95-153.

[G] Gutierrez, C., The Monge-Ampère equation, Progress in nonlinear differential equations and their applications, 44, Birkhauser, Boston, MA, 2001.

[HKM] Heinonen, J., Kilpeläinen, T. and Martio, O., Nonlinear Potential Theory of Degenerate Elliptic Equations, Oxford University Press, New York, 1993.

[J1] Jensen, R., The maximum principle for viscosity solutions of second order fully nonlinear partial differential equations, Arch. Rat. Mech. Analysis 101 (1988), 1-27.

[J2] Jensen, R., Uniqueness of Lipschitz Extensions: Minimizing the Sup Norm of the Gradient, Arch. Rational Mech. Anal. 123 (1993), pp. 51-74.

[JLM] Juutinen, P., Lindqvist, P. and Manfredi, J., On the equivalence of viscosity solutions and weak solutions for a quasi-linear equation, SIAM J. of Math. Anal. 33(3), pp. 699-717, 2001.

[L1] Lu, G., Embedding theorems into Lipschitz and BMO spaces and applications to quasilinear subelliptic differential equations, Publicacions Matemátiques, 40 (1996), 301-329.

[L2] Lu, G., Weighted Poincaré and Sobolev inequalities for vector fields satisfying Hörmander's condition and applications, Revista Matematica Iberoamericana, 8(3), 1992, 367-439.

[LM] Lindqvist,P. and Manfredi, J. ,The Harnack inequality for $\infty$-harmonic functions, Electronic Journal of Differential Equations 44, 1995.

[LM2] Lindqvist,P. and Manfredi, J. ,Note on $\infty$-harmonic functions, Revista Matemática de la Universidad Complutense 10(2), pp. 471-480, 1997.

[LMS] Lindqvist, P., Manfredi, J., and Saksman, E., Superharmonicity of nonlinear ground states, Rev. Mat. Iberoamericana, 16(2000), 17-28.

[M] Manfredi, J. Fully Nonlinear Subelliptic Equations, available at http://www.pitt.edu/ ${ }^{\text {manfredi/quasi.html. }}$

[LU] Lanconelli, E. and Uguzzoni, F., On the Poisson kernel for the Kohn Laplacian, Rend. Mat. Appl. (7) 17 (1997), no. 4, 659-677, 1998. 
[MSt] Manfredi, J. and Stroffolini B., A Version of the Hopf-Lax Formula in the Heisenberg Group, Comm. in Partial Differential Equations, 27, (2002), 1139-1159.

[MSc] Monti, R. and Serra Cassano, F., Surface measures in Carnot-Carathéodory spaces, ${ }^{*}$ Calc. Var. Partial Differential Equations 13 (2001), no. 3, 339-376.

[NSW] Nagel, A., Stein, E. and Wainger, S., Balls and metrics defined by vector fields I: Basic Properties, Acta Mathematica 55(1985), pp. 103-147.

Department of Mathematics, Wayne State University, Detroit, Mi 48202, USA

E-mail address: gzlu@math.wayne.edu

Department of Mathematics, University of Pittsburgh, Pittsburgh, PA 15260, USA

E-mail address: manfredi@pitt.edu

Dipartimento di Matematica e Applicazioni, Università degli studi di Napoli Federico II, Complesso Monte S. Angelo Edificio "T", Via Cintia, 80126 Napoli, Italy

E-mail address: stroffol@matna2.dma.unina.it 